\title{
Application of an Improved Grey Neural Network in Grain Yield Prediction
}

\author{
H. Lv, T. Lei \\ Tian Yun Rongchuang Data Technology Co. Ltd. \\ Cloud Valley Yizhuang, Daxing District \\ Beijing, China
}

\author{
X. L. Huang \\ Communication University of China \\ China
}

\author{
Y. K. Zhang \\ Jiamusi Measurment and Control Station of China Xian Satellite Control Center
}

China

\begin{abstract}
Grain yield presents a complex nonlinear relationship with variables, and takes on randomness and mutability. It is hard to describe the prediction model with traditional linear model. Grey model can be used to process samples with great stochastic volatility. In this paper, we propose to use the grey neural network to predict the grain yield. Experiments are carried out on the beans yield, rice yield and corn yield respectively to evaluate the prediction performance. The promising experimental results validate the effectiveness of our prediction model.
\end{abstract}

Keywords-grey neural network; grey model; grain yield prediction

\section{INTRODUCTION}

Traditional prediction model [1,2] assumes that the distribution of grain yield following linear variation rules, however, in fact that the grain yield presents a complex nonlinear relationship with variables such as policy factors, climate, pests diseases, food prices, etc. [3,4], and takes on randomness and mutability. It is hard to describe the prediction model with traditional linear model. The formation process of grain yield can be seen as a dynamic grey system which contains both known information and unknown information. The grey prediction method is suitable for data prediction with exponential growth only, and when the simply Grey Model (GM) used for the data with great stochastic volatility, it could get poor fit performance and lower prediction accuracy [5-7]. Neural networks $[8,9]$ can approximate the non-linear relationship between the input data and output data infinitely, and is adaptive to the prediction problem with the data of nonlinear and great stochastic volatility. It widely used in the field of industrial and agricultural production forecasting. So grey prediction model and neural networks can be combined to complement each other, constitute a grey neural network, increase the grain yield prediction accuracy.

In this paper, we apply the grey neural network to predict the grain yield, and evaluate the effectiveness of the prediction model by experiments. This paper is organized in this way: Section 2 gives a detailed description about the grey neural network and its application on grain yield; Section 3 presents experimental data and results. Finally, concluding remarks are given in Section 4.

\section{GREY NEURAL NETWORK MODEL}

Grey system theory studies uncertainty system which has small data, poor information [11]. The valuable information is extracted from the generation and development of partial known information, the behavior and evolution rule of the uncertainty system could be described properly and controlled effectively. The grey theory was proposed by Deng Julong $[12,13]$, and it considered that any random process could be seen as a varied grey process within a certain time and space, while a erratic sequence of the system could be transformed into a regular sequence by generation transform.

\section{A. Grey Model with Time Sequence}

Grey system built GM, which is a differential equation based on original data. The most typical GM is based on time sequence, it transforms the time sequence data to a differential equation and uses the system information to quantize the abstract model, finally predicts the system output in the lack of the system features.

The original data array is accumulated to generate a certain regular pattern at first, then the typical curve could be used to fit the required curve. Assume that the time data array $x^{(0)}$ can be described by the following formula:

$$
x^{(0)}=\left(x_{t}^{(0)} \mid t=1,2, \ldots, n\right)=\left(x_{1}^{(0)}, x_{2}^{(0)}, \ldots, x_{n}^{(0)}\right)
$$

Accumulate $x^{(0)}$ to generate the new time data array $x^{(1)}$, the new array $x^{(1)}$ 's $t$ item is the sum up of the first $t$ items of the original array $x^{(0)}$ as follows:

$$
x^{(1)}=\left(x_{t}^{(1)} \mid t=1,2, \ldots, n\right)=\left(\sum_{t=1}^{1} x_{t}^{(0)}, \sum_{t=1}^{2} x_{t}^{(0)}, \ldots, \sum_{t=1}^{n} x_{t}^{(0)}\right)
$$

According to the new array $x^{(1)}$, the whitening equation \[ \frac{d x^{(1)}}{d t}+a x^{(1)}=u \]
could be build as , and the solution of the
equation is $x_{t}^{*(1)}=\left(x_{1}^{(0)}-u / a\right) e^{-a(t-1)}+u / a$. Where $x_{t}^{*(1)}$ is the 
estimated value of $x_{t}^{(1)}$, the regression of the $x_{t}^{*(1)}$ is the estimated value of $x_{t}^{*(0)}$, that is $x_{t}^{*(0)}=x_{t}^{*(1)}-x_{t-1}^{*(1)} \quad t=2,3, \ldots$

\section{B. Grey Neural Network}

The differential equation of grey neural network with $n$ parameters can be described as follows:

$$
\frac{d y_{1}}{d t}+a y_{1}=b_{1} y_{2}+b_{2} y_{3}+\ldots+b_{n-1} y_{n}
$$

Where $y_{2}, y_{3}, \ldots, y_{n}$ are system input parameters and $y_{1}$ is the system output parameter, $a, b_{1}, b_{2}, \ldots, b_{n-1}$ are coefficients of parameters in the differential equation.

The time response of the above equation is

$$
\begin{aligned}
& \begin{aligned}
z(t)=\left(y_{1}(0)-\frac{b_{1}}{a}\right. & \left.y_{2}(t)-\frac{b_{2}}{a} y_{3}(t)-\ldots-\frac{b_{n-1}}{a} y_{n}(t)\right) e^{-a t} \\
& +\frac{b_{1}}{a} y_{2}(t)+\frac{b_{2}}{a} y_{3}(t)+\ldots+\frac{b_{n-1}}{a} y_{n}(t)
\end{aligned} \\
& \text { Assume that } d=\frac{b_{1}}{a} y_{2}(t)+\frac{b_{2}}{a} y_{3}(t)+\ldots+\frac{b_{n-1}}{a} y_{n}(t)
\end{aligned}
$$
above equation is transformed to be

$$
\begin{aligned}
z(t) & =\left(\left(y_{1}(0)-d\right) \cdot \frac{e^{-a t}}{1+e^{-a t}}+d \cdot \frac{1}{1+e^{-a t}}\right) \cdot\left(1+e^{-a t}\right) \\
& =\left(\left(y_{1}(0)-d\right)\left(1-\frac{1}{1+e^{-a t}}\right)+d \cdot \frac{1}{1+e^{-a t}}\right) \cdot\left(1+e^{-a t}\right) \\
& =\left(\left(y_{1}(0)-d\right)-y_{1}(0) \cdot \frac{1}{1+e^{-a t}}+2 d \cdot \frac{1}{1+e^{-a t}}\right) \cdot\left(1+e^{-a t}\right)
\end{aligned}
$$

According to equation 5, the mapped neural network could be built as shown in Fig. 1.

In Fig. $1, \mathrm{t}$ is the time order of input, $y_{2}(t), \ldots, y_{n}(t)$ are input parameters of network input. $w_{21}, w_{22}, \ldots, w_{2 n}, w_{31}, w_{32}, \ldots, w_{3 n}$ are weights of network, $y_{1}$ is the estimated value of network. LA, LB, LC, LD represent the four layers of the grey neural network respectively.

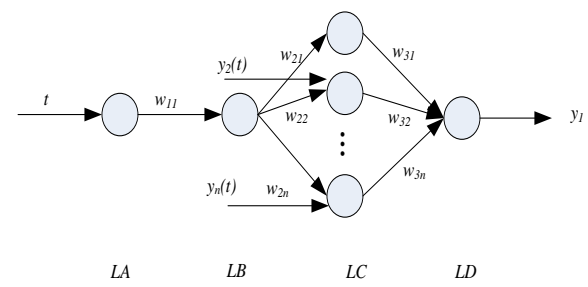

FIGURE I. GREY NEURAL NETWORK.

$$
\begin{aligned}
& \frac{2 b_{1}}{a}=u_{1}, \frac{2 b_{2}}{a}=u_{2}, \ldots, \frac{2 b_{n-1}}{a}=u_{n-1}, \text { the initial } \\
& \text { network can be presented as }
\end{aligned}
$$

$w_{11}=a, w_{21}=-y_{1}(0), w_{22}=u_{1}, w_{23}=u_{2}, \ldots, w_{2 n}=u_{n-1}$ $w_{31}=w_{32}=\ldots=w_{3 n}=1+e^{-a t}$.

The threshold of the LD output layer is $\theta=\left(1+e^{-a t}\right)\left(d-y_{1}(0)\right)$

The study process of the grey neural network is:

Step1: According to the training data to initialize the structure of network, parameters such as a,b, and use a, b to calculate $u$.

Step2: According to the definition of network weights to compute $w_{21}, w_{22}, \ldots, w_{2 n}, w_{31}, w_{32}, \ldots, w_{3 n}$.

Step3: For each input data array $(t, y(t)), t=1,2,3, \ldots, N$, calculate the output of each layer.

LA layer: $a=w_{11} t$

LB layer: $b=f\left(w_{11} t\right)=\frac{1}{1+e^{-w_{11} t}}$

LC layer:

$c_{1}=b w_{21}, c_{2}=y_{2}(t) b w_{22}, c_{3}=y_{3}(t) b w_{23}, \ldots, c_{n}=y_{n}(t) b w_{2 n}$

LD layer: $d=w_{31} c_{1}+w_{32} c_{2}+\ldots+w_{3 n} c_{n}-\theta_{y 1}$

Step4: Calculate the deviation between network prediction output and expectation output, and adjust the weights and threshold according to the deviation.

Deviation in LD layer: $\delta=d-y_{1}(t)$

Deviation in LC layer: $\delta_{1}=\delta\left(1+e^{-w_{11} t}\right), \delta_{2}=\delta\left(1+e^{-w_{11} t}\right), \ldots, \delta_{n}=\delta\left(1+e^{-w_{11} t}\right)$

Deviation in LB layer:

$$
\delta_{n+1}=\frac{1}{1+e^{-w_{11} t}}\left(1-\frac{1}{1+e^{-w_{11} t}}\right)\left(w_{21} \delta_{1}+w_{22} \delta_{2}+\ldots+w_{2 n} \delta_{n}\right)
$$

Adjust the weights according to prediction deviation. layer.

Adjust the connection weights between LB layer and LC

$$
w_{21}=-y_{1}(0), w_{22}=w_{22}-\mu_{1} \delta_{2} b, \ldots, w_{2 n}=w_{2 n}-\mu_{n-1} \delta_{n} b
$$
layer.

Adjust the connection weights between LA layer and LB

$$
w_{11}=w_{11}+a t \delta_{n+1}
$$

Adjust the threshold

$$
\theta=\left(1+e^{-w_{11} t}\right)\left(\frac{w_{22}}{2} y_{2}(t)+\frac{w_{23}}{2} y_{3}(t)+\ldots+\frac{w_{2 n}}{2} y_{n}(t)-y_{1}(0)\right)
$$


Step5: Judge if the training is finish, if not, go to step 3.

\section{Application in Grain yield Prediction}

We apply the grey neural network model into the prediction of grain yield, using the grain yields as time sequence, and other relevant factors as parameters. In the fitting processing of neural network, the in itial values of parameters are important to fitting result. In grey theory, grey correlation analysis of the grey system is used to implement modeling analysis of sequence relations by acquiring the boundary, analyzing the importance of factors and recognizing the pattern. The grey correlations between relevant variants and the grain yield are calculated as the initial coefficients value of parameters about the grey neural network.

The grey correlation degree can be calculated as follows:

Step1: Normalize the initial value of each item:

$$
X_{i}^{\prime}=X_{i} / x_{i}(1)=\left(x_{i}^{\prime}(1), x_{i}^{\prime}(2), \cdots, x_{i}^{\prime}(n)\right),(i=0,1,2, \cdots, m)
$$

Step2: Calculate the difference:

$$
\begin{gathered}
\Delta_{i}(k)=\left|x_{0}^{\prime}(k)-x_{i}^{\prime}(k)\right| \\
\Delta_{i}=\left(\Delta_{i}(1), \Delta_{i}(2), \cdots, \Delta_{i}(n)\right), \quad(i=1,2,, m)
\end{gathered}
$$

Step3: Calculate the maximu m and minimu m:

$$
\begin{gathered}
M=\max _{i} \max _{k} \Delta_{i}(k) \\
m=\min _{i} \min _{k} \Delta_{i}(k)
\end{gathered}
$$

Step4: Calculate the correlation coefficients:

$$
\gamma_{0 i}(k)=\frac{m+\xi M}{\Delta_{i}(k)+\xi M}
$$

$$
\xi \in(0,1), k=1,2, \cdots, n ; \quad i=1,2, \cdots, m
$$

Step5: Calculate the correlation degree of each item:

$$
\gamma_{0 i}=\frac{1}{n} \sum_{k=1}^{n} \gamma_{0 i}(k), \quad i=1,2, \cdots, m
$$

\section{EXPERIMENTAL RESULTS}

The experiment data was sorted from the China Statistical Abstract 2013, including total power of agricultural machinery, total power of agricultural machinery, electricity for rural use, consumption of chemical fertilizers, total area of planted and the yield from 1990 to 2012 [10]. Experiments are carried out on three kinds of grain including corn, rice and beans. The accuracy of the prediction model is evaluated by the average error rate:

$$
\text { errorRate }=\frac{|A Y-P Y|}{A Y} \times 100 \%
$$

Where AY is the actual yield and PY is the predicted yield which is acquired by the grey neural network prediction model.

We compare our methods Gray Neural Network (GNN) with three different methods to show the effective performance. The three different methods are Logistic Regression Model (LRM) [1], Gray Model (GM) [4], and Gray Model with Logistic Regression Model (LRM-GM) [14].

Experiment 1: Corn yield prediction. The data from 1991 to 2007 is used as train data, and the data from 2008 to 2013 is used as test data. The compare between the predicted yield and the actual yield is shown in Figure 2 and Table 1.

From the result, It is clearly that GNN achieves the best prediction performance with error rate $4.21 \%$, and GM get the worst prediction performance with error rate $11.82 \%$.

TABLE I. CORN YELD COMPARE.

\begin{tabular}{|c|c|c|c|c|c|c|c|}
\hline Year & 2008 & 2009 & 2010 & 2011 & 2012 & 2013 & Average Error Rate \\
\hline Actual Yield (Thousands Tons) & 165914 & 163974 & 177245 & 192781 & 205614 & 218489 & \\
\hline LRM Predicted Yield(Thousands Tons) & 157500 & 169920 & 176850 & 183900 & 196880 & 192230 & 0.0497 \\
\hline GM Predicted Yield(Thousands Tons) & 148370 & 157240 & 163600 & 172130 & 183030 & 195120 & 0.1182 \\
\hline LRM-GM Predicted Yield(Thousands Tons) & 149600 & 158970 & 165400 & 173980 & 185070 & 196680 & 0.0822 \\
\hline GNN Predicted Yield(Thousands Tons) & 168070 & 174700 & 181680 & 188520 & 196130 & 200750 & 0.0421 \\
\hline
\end{tabular}

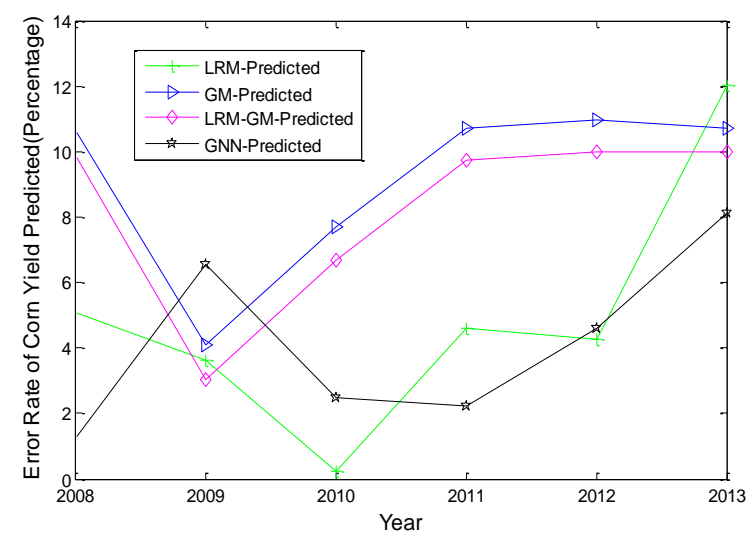

FIGURE II. ERROR RATE OF CORN YIELD PREDICTION. 
Experiment 2: Rice yield prediction. The data from 1991 to 2007 is used as train data, and the data from 2008 to 2013 is used as test data. The compare between the predicted yield and the actual yield is shown in Figure 3 and Table 2.
From Table 2, we can see that LRM achieves the best prediction performance and our method GNN get the second best prediction performance, the LRM-GM get the worst prediction performance.

TABLE II. RICE YIELD COMPARE.

\begin{tabular}{|c|c|c|c|c|c|c|c|}
\hline Year & 2008 & 2009 & 2010 & 2011 & 2012 & 2013 & Average Error Rate \\
\hline Actual Yield(Thousands Tons) & 191896 & 195103 & 195761 & 201001 & 204236 & 203612 & \\
\hline LRM Predicted Yield(Thousands Tons) & 184690 & 192730 & 195750 & 196010 & 198060 & 200130 & 0.0203 \\
\hline GM Predicted Yield(Thousands Tons) & 179250 & 181590 & 184230 & 186570 & 189600 & 192780 & 0.0651 \\
\hline LRM - GM Predicted Yield(Thousands Tons) & 177360 & 180350 & 182980 & 184920 & 188030 & 191320 & 0.0727 \\
\hline GNN Predicted Yield(Thousands Tons) & 187790 & 189340 & 190420 & 191680 & 193660 & 194750 & 0.0366 \\
\hline
\end{tabular}

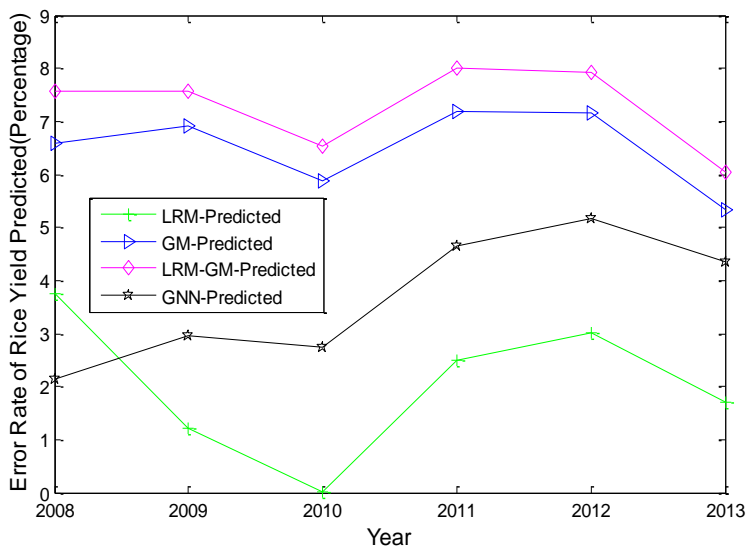

FIGURE III. ERROR RATE OF RICE YIELD PREDICTION.

Experiment 3: Beans yield prediction. The data from 1991 to 2007 is used as train data, and the data from 2008 to 2013 is used as test data. The compare between the predicted yield and the actual yield is shown in Figure 4 and Table 3.

TABLE III. BEANS YIELD COMPARE.

\begin{tabular}{|c|c|c|c|c|c|c|c|}
\hline Year & 2008 & 2009 & 2010 & 2011 & 2012 & 2013 & Average Error Rate \\
\hline Actual Yield(Thousands Tons) & 20433 & 19303 & 18965 & 19084 & 17305 & 17988 & \\
\hline LRM Predicted Yield(Thousands Tons) & 18841 & 19065 & 17646 & 16700 & 15933 & 18050 & 0.0613 \\
\hline GM Predicted Yield(Thousands Tons) & 21570 & 21547 & 21258 & 20947 & 20715 & 20182 & 0.1182 \\
\hline LRM - GM Predicted Yield(Thousands Tons) & 20836 & 20882 & 20515 & 20187 & 19998 & 19370 & 0.0789 \\
\hline GNN Predicted Yield(Thousands Tons) & 20057 & 20012 & 19393 & 18858 & 18157 & 19088 & 0.0333 \\
\hline
\end{tabular}

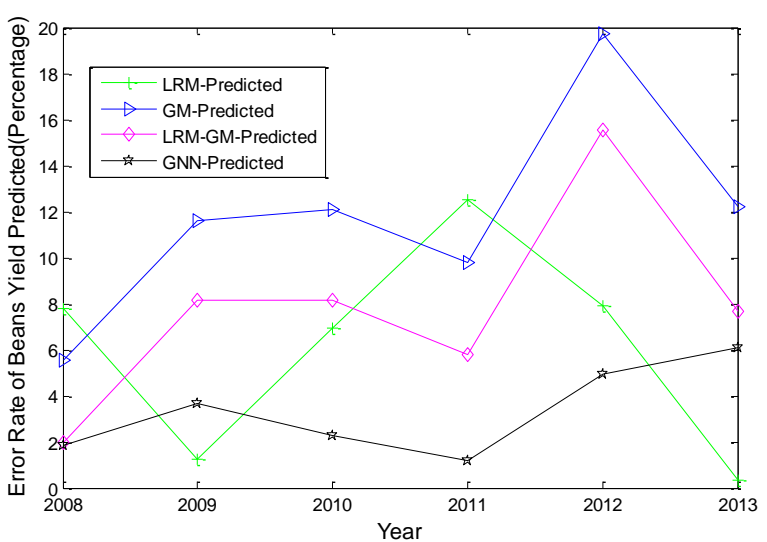

FIGURE IV. ERROR RATE OF BEANS YIELDPREDICTION.

Table 3 and Figure 4 show that our GNN method acquires the best prediction performance with error rate $3.33 \%$, and the
GM prediction method gets the worst performance with error rate $11.82 \%$.

\section{CONCLUSIONS}

We apply the grey neural network on the grain yield prediction, use the differential equation of gray model to describe the time series and map the equation on neural network to fit the equation with parameters. From the above three experiments, we can see that our proposed GNN method achieves two best prediction performance in three of them, we can conclude that our proposed GNN method improved the yield prediction performance.

\section{REFERENCES}

[1] Y. S. Zhou, Y. H. Xiao, R. S. Huang. Forecast for the grainyield of Guangxi based on the multiple regression model. Joumal of Southem Agriculture. 42(9), pp.1165-1167, 2011.

[2] M. G. Jin, C. C. Jin. Forecasting agricultural production via generalized regression neural network. In 2008 IEEE Symposium on Advanced 
Management of Information for Globalized Enterprises (AMIGE), pp. 1 3, 2008.

[3] H. H. Li. A prediction and influence factor analysis of China's food production. Information Management, Innovation Management and Industrial Engineering (ICIII),2010 3rd International Conference on, pp. 30-33, 2010.

[4] Y. N. Ren, L. Xi, Q. Wang, X. M. Ma. Application and simulation research on grain yield prediction model. Computer Simulation, 4. 8(4). pp. 208-211, 2011.

[5] Zeidenberg, Matthew. Neural Networks in Artificial Intelligence. Ellis Horwood Limited. 1990.

[6] F. Lin. Application of grey Model and Neural Network in Grain Production Prediction. Computer simulation. 29(4). pp.225-228, 2012.

[7] Y. Lin, Z. J. Teng. Prediciton of grain yield based on spiking neural networks model. 2011 IEEE 3rd International conference on Communication soft ware and Networks. pp. 171-174, 2011.

[8] Z. L. Wang; B. B. Li, L. Gao. Application of BP Neural Network Based on Principal Component Analysis in Grain Yield Prediction. 2010 2nd International Conference on information science and engineering. pp. $1011-1013,2010$

[9] H. Q. Hu, D. Zhang, Q. P. Wang. Application of Trigonometric grey prediction approach to forcasting China grain yield. Proceedings of 2009 IEEE International Conference on grey Systems and Intelligent Services, November 10-12, Nanjing, China, 2009.

[10] China Statistical Yearbook.www.stats.gov.cn/tjsj/ndsj/2013/indexch.htm (archived on 10 Oct. 2014)

[11] S. F. Liu, Y. J. Yang, L. F. Wu. In Grey System Theory and Application. 6th ed.; Science Press, China. Chapter 1.

[12] J. L. Deng. Control problems of grey systems. Systems \& Control Letters, 1(5), pp. 288-294, 1982.

[13] J. L. Deng. Introduction to grey system theory. The Journal of grey system, 1(1): pp. 1-24, 1989.

[14] J. C. Li, G. H. Chen, X. F. Shi. Grain yield forecasting based on grey multivariable linear regression. Journal of Anhui Agri. Sci., 8(16), pp. $8281-8282,2010$. 